\title{
Does the RECOVERY dexamethasone improve COVID-19 mortality?
}

\author{
Mina Kelleni ${ }^{1}$ \\ ${ }^{1}$ Affiliation not available
}

December 1, 2020

\begin{abstract}
On the same day the results of the RECOVERY study was published; July 17, 2020, we have submitted a letter to New England Journal of Medicine (NEJM, ID 20-25534) representing some concerns about this study and its status was with editor until November 29, 2020 on which a withdrawal request has been sent to NEJM and was promptly accepted on the following day. Notably, four emails were sent to NEJM, once every month wondering about this unprecedent delay in publishing a short letter and the response was that the editor has been contacted on multiple occasions but he/she is busy because of the large number of COVID-19 related submissions.
\end{abstract}

Correspondence

Does the RECOVERY dexamethasone improve COVID-19 mortality?

Mina T. Kelleni, MD, PhD

Assistant Professor of Pharmacology, College of Medicine, Minia University, Egypt.

$+201200382422$

mina.kelleni@mu.edu.eg, drthabetpharm@yahoo.com

The randomized open-label evaluation of COVID-19 therapy (RECOVERY) collaborative group showed a small mortality benefit to dexamethasone therapy among patients who were receiving invasive mechanical ventilation; 482/2104 patients $(22.9 \%)$ in the dexamethasone group and $1110 / 4321$ patients $(25.7 \%)$ in the usual care group ${ }^{1}$, yet several concerns regarding design of the study, the data and its interpretation were $\operatorname{raised}^{2,3}$. We would like to add that on the same day the results of RECOVERY study was published; July 17, 2020, we have submitted a letter to New England Journal of Medicine (NEJM, ID 20-25534) representing some concerns about this study and its status was with editor until November 29, 2020 on which a withdrawal request has been sent to NEJM and was promptly accepted on the following day. Notably, four emails were sent to NEJM, once every month wondering about this unprecedent delay in publishing a short letter and the response was that the editor has been contacted on multiple occasions but he/she is busy because of the large number of COVID-19 related submissions. Our concerns were a wonder about the reason that the RECOVERY group chose 2:1 randomization though dexamethasone is a cheap drug and a 1:1 randomization maximizes the statistical power to detect the same difference ${ }^{4,5}$. Further, in Table 2, death included in secondary outcomes was similar in ratio; $21.7 \%$ in dexamethasone group to $22.7 \%$ in the usual care group and this finding was suggested to be explained thoroughly together with more clarification to the numbers included e.g. the number of living patients who have not been discharged from hospital within 28 days doesn't match the subsequent subgroups. Furthermore, a second randomization of $4.5 \%$ of patients, complaining of hypoxia and inflammation, in the dexamethasone arm to compare the addition of tocilizumab on top of dexamethasone has not been excluded in interpretation of the benefit assumed to be attributed 
only to dexamethasone. Finally, we would like to agree with the clinical recommendation against the routine use of glucocorticoids in the management of COVID-19 especially after important concerns were raised regarding the interpretation of data as well as the limitations of several studies showing potential benefit of glucocorticoids in COVID- $19^{6}$. Moreover, a potential real benefit from the RECOVERY dexamethasone in selected COVID-19 critical cases should be confirmed or denied by other well designed and interpreted double blind large clinical trials. Another ethical call for all journals, which publish any COVID-19 clinical trial, to adhere to a transparent policy that obligates an urgent handling of any related criticism might also be considered in order not to see another short letter remaining more than four months with the busy editor.

Acknowledgment

The author would like to thank NEJM and its editor in Chief; Dr. Eric Rubin for the prompt rejection to publish this perspective (NEJM ID: 20-34248).

Conflict of interests

None

Funding

None

References:

1. Dexamethasone in Hospitalized Patients with Covid-19 - Preliminary Report. New England Journal of Medicine. 2020.

2. Waterer GW, Rello J. Steroids and COVID-19: We Need a Precision Approach, Not One Size Fits All. Infect Dis Ther.2020;9(4):701-705.

3. Lanthier L, Mayette M, Huard G, Plourde ME, Cauchon M. [In patients hospitalized for COVID19, does dexamethasone reduce 28-days mortality compared to standard treatment?]. Rev Med Interne.2020;41(11):790-791.

4. Torgerson D, Campbell M. Unequal randomisation can improve the economic efficiency of clinical trials. J Health Serv Res Policy.1997;2(2):81-85.

5. Hey SP, Kimmelman J. The questionable use of unequal allocation in confirmatory trials. Neurology. 2014;82(1):77-79.

6. Kelleni MT. Early use of non-steroidal anti-inflammatory drugs in COVID-19 might reverse pathogenesis, prevent complications and improve clinical outcomes. Biomedicine $\mathscr{E}$ Pharmacotherapy.2021;133:110982110982. 